\title{
p53-Dependent ICAM-1 overexpression in senescent human cells identified in atherosclerotic lesions
}

\author{
Vassilis G Gorgoulis ${ }^{1, *}$, Harris Pratsinis ${ }^{2, *}$, Panayotis Zacharatos ${ }^{1}$, Catherine Demoliou ${ }^{3}$, \\ Fragiska Sigala ${ }^{4}$, Panayiotis J Asimacopoulos ${ }^{5}$, Athanasios G Papavassiliou ${ }^{6}$ \\ and Dimitris Kletsas ${ }^{2, *}$
}

${ }^{1}$ Department of Histology and Embryology, Molecular Carcinogenesis Group, Medical School, University of Athens, Athens, Greece; 'aboratory of Cell Proliferation and Ageing, Institute of Biology, NCSR 'Demokritos', Athens, Greece; ${ }^{3}$ The Cyprus Institute of Neurology and Genetics, Nicosia, Cyprus; ${ }^{4}$ Department of Vascular and Endovascular Surgery, St Josef Hospital, Germany; ${ }^{5}$ Baylor College of Medicine, Houston, TX, USA and ${ }^{6}$ Department of Biochemistry, School of Medicine, University of Patras, Patras, Greece

\begin{abstract}
Most normal somatic cells enter a state called replicative senescence after a certain number of divisions, characterized by irreversible growth arrest. Moreover, they express a pronounced inflammatory phenotype that could contribute to the aging process and the development of age-related pathologies. Among the molecules involved in the inflammatory response that are overexpressed in senescent cells and aged tissues is intercellular adhesion molecule-1 (ICAM-1). Furthermore, ICAM-1 is overexpressed in atherosclerosis, an agerelated, chronic inflammatory disease. We have recently reported that the transcriptional activator p53 can trigger ICAM-1 expression in an nuclear factor-kappa B (NF- $\kappa$ B)-independent manner (Gorgoulis et al, EMBO J. 2003; 22: 1567-1578). As p53 exhibits an increased transcriptional activity in senescent cells, we investigated whether p53 activation is responsible for the senescence-associated ICAM-1 overexpression. To this end, we used two model systems of cellular senescence: (a) human fibroblasts and (b) conditionally immortalized human vascular smooth muscle cells. Here, we present evidence from both cell systems to support a p53mediated ICAM-1 overexpression in senescent cells that is independent of NF- $\kappa \mathrm{B}$. We also demonstrate in atherosclerotic lesions the presence of cells coexpressing activated p53, ICAM-1, and stained with the senescence-associated $\beta$-galactosidase, a biomarker of replicative senescence. Collectively, our data suggest a direct functional link between p53 and ICAM-1 in senescence and age-related disorders.
\end{abstract}

Laboratory Investigation (2005) 85, 502-511, advance online publication, 14 February 2005; doi:10.1038/labinvest.3700241

Keywords: aging; atherosclerosis; fibroblasts; ICAM-1; p53; smooth muscle cells

Most normal human cells do not divide indefinitely. In contrast, they exhibit a limited proliferative potential. When cultured in vitro they can undergo only a finite number of cell divisions before entering a nondividing state, referred to as replicative senescence, ${ }^{1-3}$ which has been suggested to potentially contribute to the aging process and the development of age-related diseases. ${ }^{1}$ The presence of senescent cells in tissues remained for

Correspondence: Dr D Kletsas, PhD, Laboratory of Cell Proliferation and Ageing, Institute of Biology, NCSR 'Demokritos', 15310 Athens, Greece.

E-mail: dkletsas@bio.demokritos.gr

*These authors contributed equally to this work.

Received 29 July 2004; revised 26 November 2004; accepted 30 November 2004; published online 14 February 2005 long a matter of debate. Recently, however, a senescence-associated biomarker (senescenceassociated $\beta$-galactosidase staining-SA- $\beta$-Gal) has been described, that allows the identification of senescent cells not only in culture, but also in several tissues with increasing age or in age-related disorders. $^{4-6}$ Besides the loss of proliferative capacity, replicative senescence is accompanied by a series of specific alterations in cellular morphology and function. ${ }^{3}$ Changes in gene expression patterns indicate that senescent cells are characterized by a less fibrogenic and a pronounced inflammatory phenotype, ${ }^{7,8}$ which could be responsible for age-related alterations in vivo. However, the mechanism underlying the connection between the senescent state and inflammation awaits elucidation. 
Among the molecules involved in the inflammatory response that are overexpressed in senescent cells and aged tissues is intercellular adhesion molecule-1 (ICAM-1 or CD54) ${ }^{6,7}$ ICAM-1 is a member of the immunoglobulin gene superfamily, which binds to the $\beta 2$ leukocyte integrins, leukocyte function antigen-1 (LFA-1, CD11a/CD18), Mac-1 (CD11b/CD18), and is also expressed in other cells including antigen-presenting cells, where it functions as a costimulatory molecule for T-cell activation (reviewed in Cotran and Mayadas-Norton ${ }^{9}$ ). Its importance is evident from the phenotype of ICAM$1^{-l-}$ mice that exhibit several immune defects. ${ }^{9}$ Furthermore, in humans, it is overexpressed in atherosclerosis, an age-related, chronic inflammatory disease. ${ }^{10}$ ICAM-1 is induced by several cytokines and stress stimuli such as hypoxia, ultraviolet and ionizing radiation ${ }^{11-14}$ and the nuclear factor-kappa B (NF- $\kappa \mathrm{B})$ signalling cascade plays an important role in its activation. ${ }^{15}$ However, several reports indicate that $\mathrm{NF}-\kappa \mathrm{B}$-independent pathways may also participate in ICAM-1 stimulation. ${ }^{16-18}$ In this vein, we have recently demonstrated that the transcriptional activator p53 can trigger ICAM-1 expression in an NF- $\kappa$ B-independent manner. ${ }^{19}$ This induction is abolished in the presence of the specific p53 inhibitor pifithrin- $\alpha$ and is abrogated in p53-deficient cell lines. In addition, we have shown that p53 most probably mediates its effect on ICAM-1 directly by binding on two p53-responsive elements to the introns of ICAM-1 gene. ${ }^{19}$

In senescent cells, p53 exhibits an increased DNAbinding and transcriptional activity and, when overexpressed, it can induce a senescent-like phenotype. ${ }^{20}$ Moreover, mice that express a modestly hyperactive p53 form display a premature onset of a spectrum of age-related features and reduced longevity, hence implicating p53 also in organismal aging. ${ }^{21}$ Bearing in mind that ICAM-1 is overexpressed in senescent cells and aged tissues, $, 7,22$ we explored whether p53 activation is responsible for the overexpression of ICAM-1 in cellular senescence. Furthermore, this hypothesis was addressed in a classical age-related disorder, such as atherosclerosis. ${ }^{6}$

\section{Materials and methods}

\section{Cells and Culture Conditions}

Human fetal foreskin fibroblasts (strain $\mathrm{HFFF}_{2}$ ) were obtained from the European Collection of Cell Cultures (ECACC, Salisbury, UK), and they were routinely cultured in minimum essential medium (MEM) supplemented with penicillin $(100 \mathrm{U} / \mathrm{ml})$, streptomycin $(100 \mu \mathrm{g} / \mathrm{ml})$ and $10 \%$ fetal bovine serum (all media and antibiotics from Biochrom KG, Berlin, Germany). The cells were serially subcultured using trypsin-citrate $(0.25-0.3 \%$, respectively) until they reached proliferative senescence; the number of cumulative population doublings (CPDs) achieved was recorded as previously described. ${ }^{23}$

The conditionally immortalized human vascular smooth muscle cell line HVTs-SM1 was previously developed in our laboratories. ${ }^{24}$ The cells were routinely cultured at the permissive temperature of $36^{\circ} \mathrm{C}$ in Dulbecco's modified Eagle's medium (DMEM) supplemented with neomycin (G418 $200 \mu \mathrm{g} / \mathrm{ml}$, Gibco-Invitrogen, Paisley, UK), as well as, penicillin $(100 \mathrm{U} / \mathrm{ml})$, streptomycin $(100 \mu \mathrm{g} / \mathrm{ml})$ and $10 \%$ fetal bovine serum. HVTs-SM1 cells when being at approx. $80-90 \%$ confluency were subcultured with trypsin-citrate, as described above. Senescence was induced by shifting HTVs-SM1 cultures to the nonpermissive temperature of $39^{\circ} \mathrm{C}^{24,25}$

For both cell types, cellular senescence was identified microscopically by their altered morphology and their inability to proliferate, and was further confirmed by using bromodeoxyuridine labelling, as well as $\mathrm{p} 21^{\mathrm{WAF} 1}$ expression and SA- $\beta$-Gal staining.

\section{Effect of Specific Inhibitors and of TNF- $\alpha$ on ICAM-1 Expression}

Based on our previous findings, ${ }^{19}$ we have studied the effect of p53 and NF- $\kappa$ B on ICAM-1 expression in senescent cells. To this end, the cultures were treated with the specific inhibitors pifithrin- $\alpha$ hydrobromide (PFT- $\alpha$-Tocris, Ellisville, MO, USA $)^{19,26}$ at the indicated concentrations or BAY 11-7082 (Sigma, St Louis, MO, USA) ${ }^{27}$ at $10 \mu \mathrm{M}$, respectively, and for the indicated time-points. Finally, ICAM-1 expression was also measured $24 \mathrm{~h}$ after the addition of tumor necrosis factor- $\alpha$ (TNF- $\alpha-R \& D$, Systems, Minneapolis, MN, USA) (10 ng/ml).

\section{Tissue Specimens}

A total of 20 atherosclerotic plaques of human carotid arteries were obtained from patients undergoing carotid endarterectomy. The lesions were type V (fibroatheroma) and VI (complicated) according to the American Heart Association classification. ${ }^{28}$ Two samples of each plaque were obtained: one washed with PBS and immersed in SA- $\beta$-Gal staining solution (see below) and the other one fixed in $4 \%$ buffered paraformaldeyde, embedded in paraffin (FFPE) and prepared according to standard methods. Serial sections were stained for hematoxylin and eosin, and used for immunohistochemistry. Moreover, three histologically normal internal mammary arteries from patients undergoing aorto-coronary bypass surgery were included as control tissue.

\section{SA- $\beta$-Gal Staining}

SA- $\beta$-Gal staining in cells and tissues was performed as described by Dimri et al, ${ }^{4}$ modified as follows:

Cell cultures were fixed for $5 \mathrm{~min}$ in neutral formalin (approx. $4 \%$ formaldehyde), washed in 
PBS and incubated for $12-24 \mathrm{~h}$ at $37^{\circ} \mathrm{C}$ with $1 \mathrm{mg} / \mathrm{ml}$ $\mathrm{X}$-Gal (5-bromo-4-chloro-3-indolyl $\beta$-D-galactoside, Sigma) in a buffer containing $40 \mathrm{mM}$ citric acid/ sodium phosphate $\mathrm{pH}: 6.0,5 \mathrm{mM}$ potassium ferrocyanide, $5 \mathrm{mM}$ potassium ferricyanide, $150 \mathrm{mM}$ sodium chloride and $2 \mathrm{mM}$ magnesium chloride (SA- $\beta$-Gal staining solution).

Carotid plaques were incubated for $24 \mathrm{~h}$ at $37^{\circ} \mathrm{C}$ in the SA- $\beta$-Gal staining solution (see above). After incubation, the tissue was fixed in $4 \%$ paraformaldehyde, embedded in paraffin and treated as FFPE material.

\section{mRNA Analysis}

The mRNA levels of the studied genes were assessed using a comparative multiplex RT-PCR method, as previously described. ${ }^{19}$ Briefly, RNA was extracted using the GenElute extraction kit (Sigma) and subsequently cDNA was generated using the M-MLV Superscript II RT according to the manufacturer's instructions (Life Technologies-Invitrogen). The GAPDH gene was used as reference gene for all PCRs. The following amplimers were designed using the Oligo 4.01 software (National Biosciences Inc., Plymouth, MN, USA): GAPDH (Accession No. XM_033263) forward (F), CAT CTC TGC CCC CTC TGC TG (position 830), reverse (R), CGA CGC CTG CTT CAC CAC CT (position 411), product length $438 \mathrm{bp}$; p21 ${ }^{\mathrm{WAF} 1}$ (Accession No. U03106) F, CTG CCG CCG CCT CTT C (position 126), R, CTG AGC GAG GCA CAA GGG TA (position 426), product length $319 \mathrm{bp}$, annealing temperature $61^{\circ} \mathrm{C}$, plus $5 \%$ dimethylsulfoxide (DMSO); ICAM-1 (Accession No. X06990) F, TGG TAG CAG CCG CAG TCA TA (position 1469), R, CTC CTT CCT CTT GGC TTA GT (position 1829), product length $377 \mathrm{bp}$, annealing temperature $57^{\circ} \mathrm{C}$. PCR products were electrophoresed in a nondenaturing $8 \%$ acrylamide/ bis-acrylamide (19:1) gel. Gels were stained with ethidium bromide and images were captured with a CCD camera connected to a PC, using the BioProfil gel documentation software (Vilber Lourmat, TorcyParis, France).

\section{Western Immunoblot Analysis}

For the collection of whole cell-lysates, the cellmonolayers were washed with ice-cold tris buffered saline (TBS: $10 \mathrm{mM}$ Tris-HCl pH 7.4, $150 \mathrm{mM} \mathrm{NaCl}$ ) and scraped immediately in hot SDS-PAGE sample buffer, that is, $62.5 \mathrm{mM}$ Tris $\mathrm{pH} 6.8,6 \% \mathrm{w} / \mathrm{v}$ SDS, $2 \% \mathrm{v} / \mathrm{v}$ glycerol, $5 \% \mathrm{v} / \mathrm{v} 2$-mercaptoethanol, 0.0125 $\% \mathrm{w} / \mathrm{v}$ bromophenol blue, protease- and phosphatase-inhibitor cocktails (Sigma). Following sonication for $15 \mathrm{~s}$, the samples were clarified by centrifugation and stored at $-80^{\circ} \mathrm{C}$ until use.

Samples were separated on 9\% SDS-PAGE and the proteins were transferred to PVDF membranes (Amersham Biosciences, Buckinghamshire, UK).
The membranes were blocked with $5 \%$ nonfat milk in TBS containing $0.1 \%$ Tween-20 (TTBS) and incubated with the primary mouse monoclonal antibodies, that is, anti-ICAM-1 (G-5) (class IgG2a; epitope, amino acids 258-365, human origin, Santa Cruz Biotechnology, Santa Cruz, CA, USA), antip21 WAF1 (Class: IgG, epitope: residues 1-159 of p21 ${ }^{\mathrm{WAF} 1}$, Santa Cruz Biotechnology), anti- $\alpha$-tubulin (Sigma) or PanActin antibody (NeoMarkers, Lab Vision Corporation, Fremont, CA, USA). Following incubation with horseradish peroxidase-conjugated secondary anti-mouse antibody (Sigma), immunoreactive bands were visualized on Kodak-X-OMAT AR film by chemiluminescence (ECL kit) according to the manufacturer's (Amersham) instructions. The intensity of the bands was quantified as described above.

\section{Immunohistochemistry}

Antibodies

For immunohistochemical analysis, the following antibodies (Abs) were used: anti-ICAM-1 and antip21 ${ }^{\mathrm{WAF} 1}$ (see above), as well as DO7 (Class: IgG2b, mouse monoclonal, epitope: residues 1-45 of p53) (Dako, Kalifronas Athens Greece).

\section{Method}

Paraffin sections, 5- $\mu \mathrm{m}$ thick, of the lesions were mounted on poly-a-lysine-coated slides, dewaxed, rehydrated and incubated for $30 \mathrm{~min}$ with $0.3 \%$ hydrogen peroxide to quench the endogenous peroxidase activity. Unmasking of ICAM-1, p53, and $\mathrm{p} 21^{\mathrm{WAF} 1}$ proteins was carried out with the heatmediated antigen retrieval method using $1 \mathrm{mM}$ EDTA pH 8.0 in a steamer. The sections were incubated with the antibodies at a 1:100 dilution at $4{ }^{\circ} \mathrm{C}$ overnight. Biotin-conjugated secondary antibody (Dako) was added at a 1:200 dilution for $1 \mathrm{~h}$ at room temperature (RT). Strept-Avidin-Biotin complex (Dako) at a dilution of 1:100 was applied to enhance the signal. For color development, we used 3,3'-diaminobenzidine tetrahydrochloride $(\mathrm{DAB})$ and hematoxylin as counterstain.

\section{Controls}

Mouse IgG1 MAb of unrelated specificity and the IgG fraction of normal rabbit serum were used as negative controls.

\section{Results}

\section{ICAM-1 Overexpression in Senescent Human Fibroblasts is Mediated by p53}

Initially, we investigated the expression of ICAM-1 in normal human fibroblasts that have undergone senescence after serial subculturing in vitro, in comparison to young (early-passage) cells. Figure 1a depicts the typical appearance of senes- 
cent fibroblasts. The cells, in contrast to the spindlelike appearance of young fibroblasts, are highly enlarged, irregular in shape and with an intense perinuclear SA- $\beta$-Gal staining. We observed that senescent fibroblasts strongly overexpress ICAM-1, both at the mRNA and protein levels, compared to young cells. An 2.5-fold augmentation was found at the RNA levels, while at the protein level this effect was more intense: an $\sim 5$-fold increase was detected (Figure 1b, c).

Next, we used a novel low-molecular-weight compound that inhibits p53-mediated transcription, namely PFT- $\alpha$. This agent has been shown to inhibit the activation of p53-responsive genes, including $p 21^{W A F 1}$ and $m d m 2 .{ }^{26}$ PFT- $\alpha$ blocks the activation of p53 in cells after a variety of treatments, for example, doxorubicin, etoposide, Taxol, cytosine arabinoside, UV- and $\gamma$-irradiation, and there are already suggestions for potential clinical applications (for a review, see Komarova and Gudkov ${ }^{29}$ ). In addition, we have recently shown, that PFT- $\alpha$ effectively inhibits the p53-mediated ICAM-1 and $p 21^{\text {WAF1 }}$ induction after $\gamma$-irradiation of human fibroblasts. ${ }^{19}$ Here, we demonstrate that in the presence of $20 \mu \mathrm{M}$ PFT- $\alpha$, the observed ICAM-1 overexpression in senescent fibroblasts was markedly impaired (Figure 1b, c). Interestingly, PFT- $\alpha$ has the same effect on the expression of $\mathrm{p} 21^{\mathrm{WAF} 1}$, a classical p53 target gene and a marker of cellular senescence. ${ }^{3}$ As expected, p21 ${ }^{\mathrm{WAF} 1}$ was found to be

a

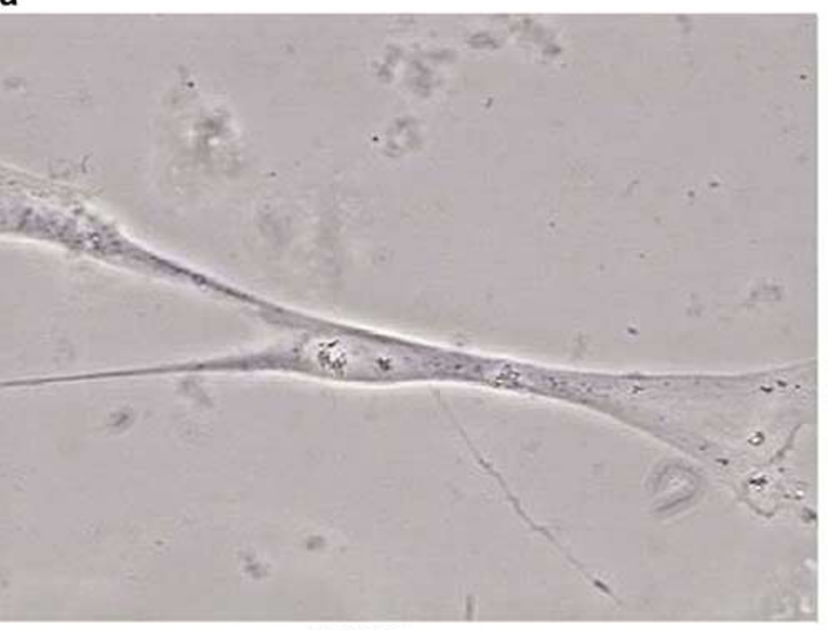

CPD 32
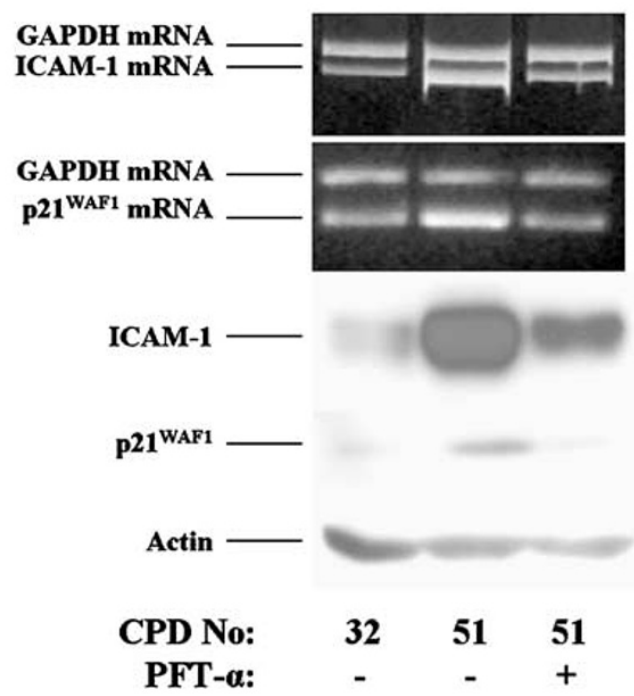

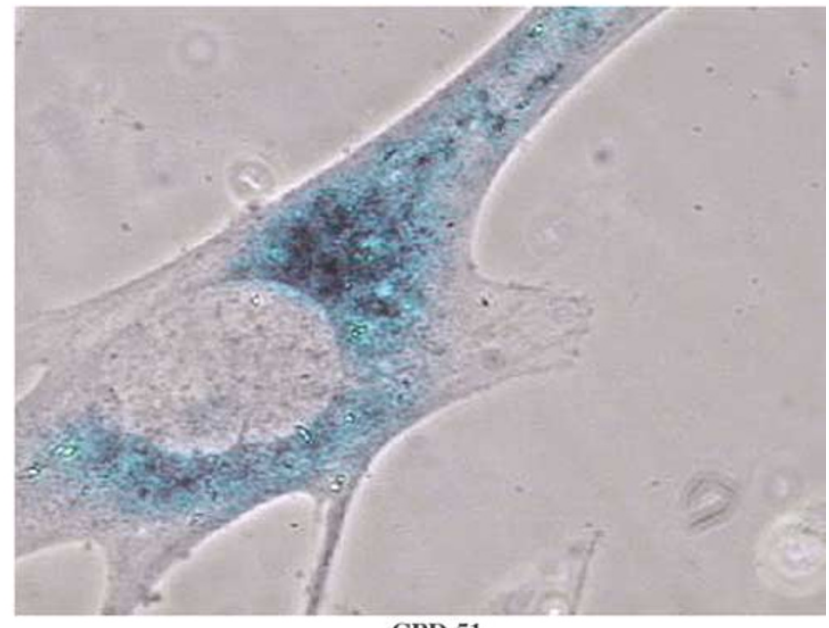

CPD 51

C
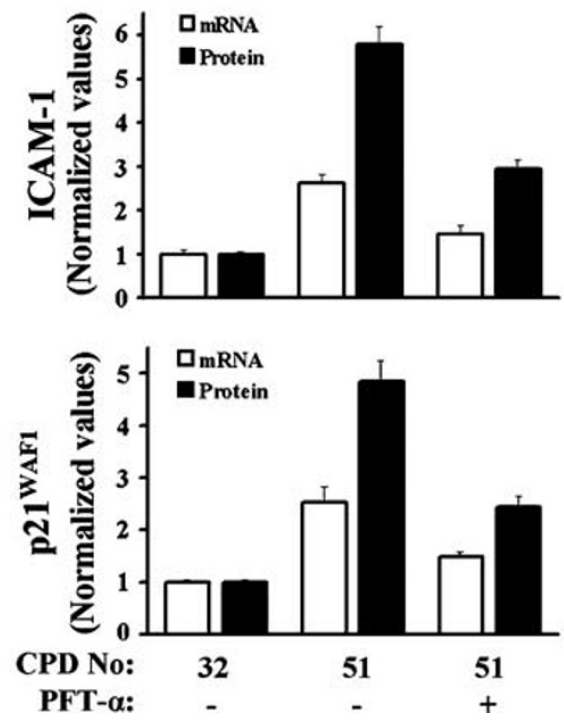

Figure 1 ICAM-1 expression in senescent human skin fibroblasts. The human fibroblast cell strain $\mathrm{HFFF}_{2}$ has been passaged until it reached replicative senescence, as described under Materials and methods. In (a) young (CPD 32) and senescent (CPD 51) cells were stained with the SA- $\beta$-Gal staining (magnification $\times 1000$ ). In (b) young and senescent cells, as well as, senescent cells treated with $20 \mu \mathrm{M}$ PFT- $\alpha$ for $12 \mathrm{~h}$ (for mRNA analysis) or $24 \mathrm{~h}$ (for protein analysis) were studied for the expression of ICAM-1 and p21 $21^{\text {WAF1 }}$ at the mRNA level by RT-PCR and at the protein level by Western analysis; GAPDH and actin levels were used as loading controls, respectively. In (c) the intensity of the bands was assessed by densitometric analysis and it is presented after normalization according to the intensity of the loading controls (mean of three experiments; error bars indicate standard deviation). 
506

a
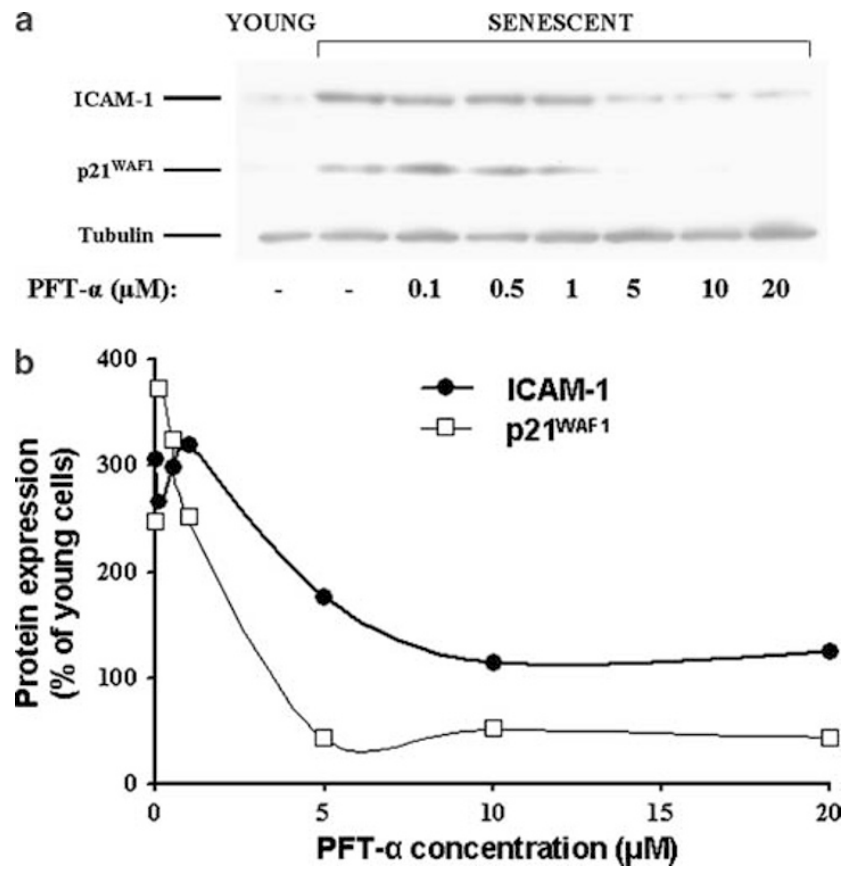

Figure 2 Dose-dependent inhibition of ICAM-1 expression in senescent human skin fibroblasts by PFT- $\alpha$. In (a) senescent human skin fibroblasts treated with the indicated concentrations of PFT- $\alpha$ for $24 \mathrm{~h}$ were studied for the expression of ICAM-1 and p $21^{\text {WAF1 }}$ by Western analysis; tubulin levels were used to ensure equal loading, while the lysate of young fibroblasts was included as control. In (b) the intensity of the bands was assessed by densitometric analysis, normalized according to the intensity of the loading controls and it is presented as a percentage of the expression levels in young cells.

overexpressed in senescent cells, at the mRNA and protein levels, and this effect was largely reversed by PFT- $\alpha$ (Figure 1b,c). Furthermore, the inhibitory effect of PFT- $\alpha$ on the overexpression of ICAM-1 in senescent human fibroblasts was found to be dosedependent: an approximately $50 \%$ inhibition was obtained with $5 \mu \mathrm{M}$ PFT- $\alpha$, while over $10 \mu \mathrm{M}$ the senescence-induced ICAM-1-overexpression was almost completely annulled (Figure 2). A similar pattern was also observed in the expression of p2 $1^{\mathrm{WAF} 1}$ in senescent cells in the presence of PFT- $\alpha$ (Figure 2).

\section{p53 Activation in a Model of Senescent Human Vascular Smooth Muscle Cells Leads to Enhanced ICAM-1 Expression}

We next studied ICAM-1 expression in senescent human smooth muscle cells (SMCs). It is well known that the in vitro study of SMCs, especially from human origin, is hindered by the fact that their responses vary considerably among donors. ${ }^{30,31}$ To circumvent this, we used an immortalized cell line that we have recently developed, designated as HVTs-SM1. This cell line was developed from human vascular SMCs, stably transfected with a nonreplicative retroviral vector containing a temperature-sensitive (tsA58) mutant of the SV40 large T-antigen. ${ }^{24}$ SV40 large T-antigen targets and inactivates pivotal cell-cycle regulators, like p53. ${ }^{32}$ Accordingly, at the permissive temperature of $36^{\circ} \mathrm{C}$, these cells can proliferate indefinitely, while at the nonpermissive temperature of $39^{\circ} \mathrm{C}$ (where large $\mathrm{T}$ antigen expression is downregulated, hence p53 is active) they acquire a senescent phenotype. ${ }^{24}$ As shown in Figure 3, HVTs-SM1 cells exhibit an intense expression of the senescence markers $\mathrm{p} 21^{\mathrm{WAF} 1}$ and SA- $\beta$-Gal staining only at the nonpermissive temperature. Senescent HVTs-SM1 cells strongly overexpress also ICAM-1, at the mRNA and protein levels. An $\sim 3$-fold increase was observed in comparison to HVTs-SM1 maintained at the permissive temperature (Figure 3b,c). Once again, in the presence of $20 \mu \mathrm{M}$ of the p53 inhibitor PFT- $\alpha$, this ICAM-1 overexpression in senescent cells was severely reduced (Figure 3b,c). An analogous expression pattern was obtained for p21 ${ }^{\mathrm{WAF} 1}$ : the overexpression observed in senescent HVTs-SM1 cells at the nonpermissive temperature, due to p53 activation, was largely reversed by PFT- $\alpha$ (Figure 3b, c).

\section{ICAM-1 Overexpression in Senescent Cells is not Mediated by the NF- $\kappa$ B Pathway}

It is well appreciated that $\mathrm{NF}-\kappa \mathrm{B}$ is the main transcriptional regulator of ICAM-1 expression. Accordingly, we examined whether the overexpression of ICAM-1 in senescent cells is also mediated through this signalling cascade. First, we studied the response of young and senescent cells to TNF- $\alpha$, as the latter is a potent stimulator of ICAM-1 expression, acting through the NF- $\kappa$ B pathway., ${ }^{9,11,15}$ Figure $4 \mathrm{a}$ reveals that TNF- $\alpha$ potentiates ICAM-1 expression significantly in both young and senescent fibroblasts and HVTs-SM1 cells, implying that the TNF- $\alpha-N F-\kappa B$ pathway is functional in these cells. Next, we employed a specific NF- $\kappa$ B inhibitor, BAY 11-7082. ${ }^{27}$ This compound, when used at a concentration of $10 \mu \mathrm{M}$, has been shown to selectively inhibit the TNF- $\alpha$-induced surface expression of ICAM-1 by blocking the phosphorylation of $\mathrm{IkB}-\alpha .{ }^{27}$ As demonstrated in Figure 4b, BAY 117082 can severely suppress the TNF- $\alpha$-mediated ICAM-1 expression in fibroblasts. Interestingly, it slightly suppresses the basal levels of ICAM-1 expression in young cells, possibly indicating that these are controlled by the NF- $\kappa \mathrm{B}$ pathway. On the other hand, PFT- $\alpha$ has no effect either to the basal or TNF- $\alpha$-induced ICAM-1 expression, indicating that it does not interfere with the TNF- $\alpha-\mathrm{NF}-\kappa \mathrm{B}$ pathway. Finally, the presence of $10 \mu \mathrm{M}$ BAY $11-7082$ was incapable of reverting ICAM-1 overexpression in both senescent human fibroblasts and senescent HVTs-SM1 cells (Figure 4c). 
a

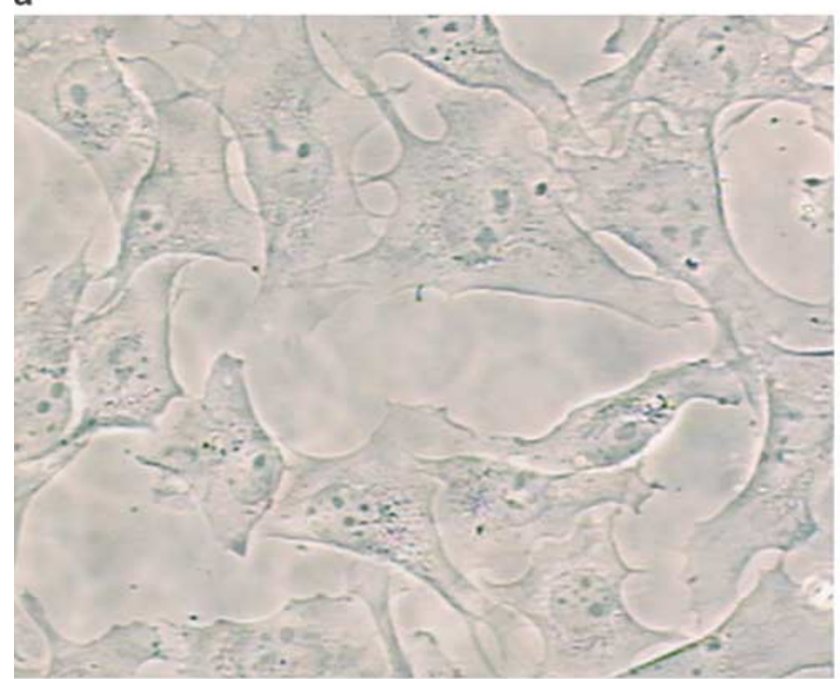

$36^{0} \mathrm{C}$

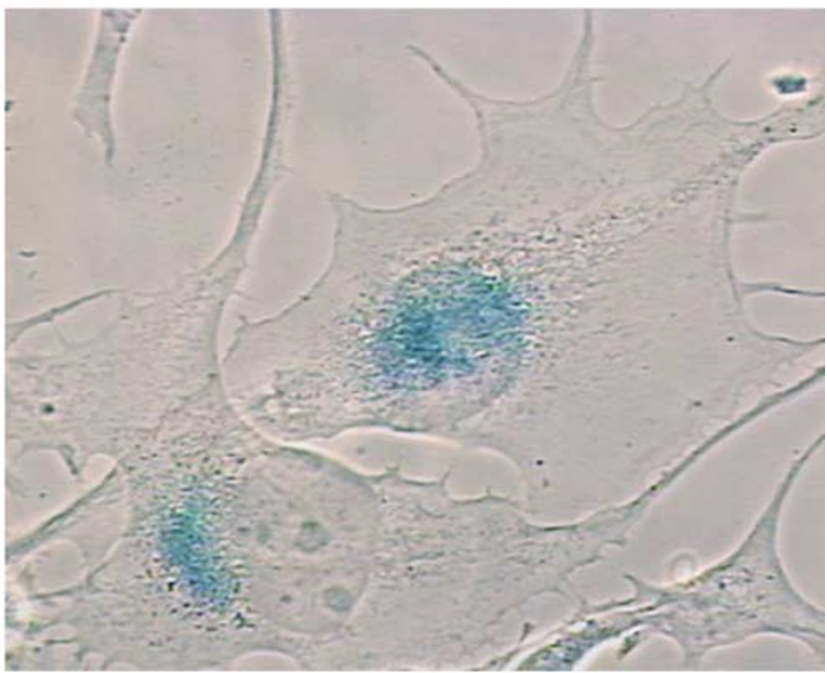

$39^{\circ} \mathrm{C}$ b

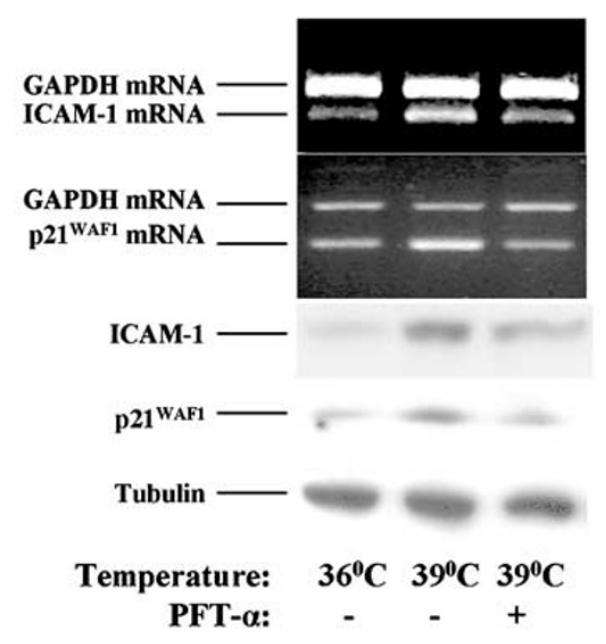

C
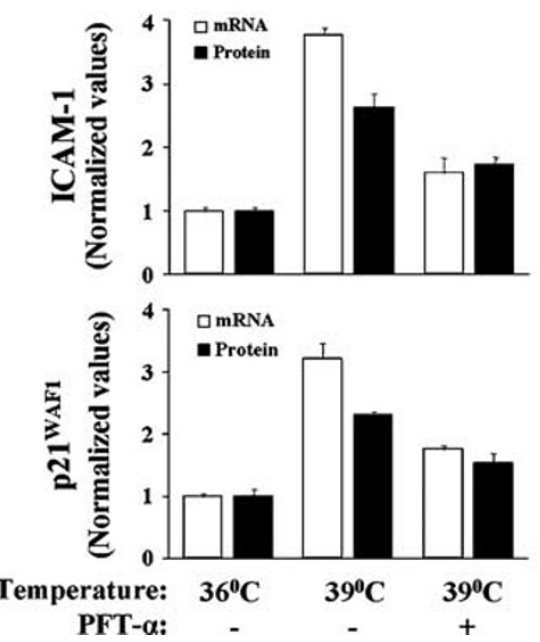

Figure 3 ICAM-1 expression in senescent human smooth muscle cells. The conditionally immortalized human vascular smooth muscle cell line HVTs-SM1 was cultured at the permissive $\left(36^{\circ} \mathrm{C}\right)$ and at the nonpermissive $\left(39^{\circ} \mathrm{C}\right)$ temperatures as described under Materials and methods. In (a) the cells were stained with the SA- $\beta$-Gal staining (magnification $\times 1000$ ). In (b) cells cultured at both permissive and nonpermissive conditions, as well as, cells at the nonpermissive temperature treated with $20 \mu \mathrm{M}$ PFT- $\alpha$ for $12 \mathrm{~h}$ (for mRNA analysis) or $24 \mathrm{~h}$ (for protein analysis) were studied for the expression of ICAM-1 and p21 ${ }^{\mathrm{WAF} 1}$ at the mRNA level by RT-PCR and at the protein level by Western analysis; GAPDH and tubulin levels were used as loading controls, respectively. In (c) the intensity of the bands was assessed by densitometric analysis and it is presented after normalization according to the intensity of the loading controls (mean of three experiments; error bars indicate standard deviation).

Overall, the results from both cell systems indicate that p53 activation in senescent cells induces ICAM-1 overexpression, in an NF- $\kappa \mathrm{B}-$ independent manner.

\section{Co-expression of p53, ICAM-1 and SA- $\beta$-Gal Activity in Atherosclerotic Lesions}

Having shown that ICAM-1 is overexpressed in in vitro senescent cells, we went on to study this phenomenon also in vivo, and in particular in a typical age-related inflammatory disease, that is, atherosclerosis. As demonstrated in Figure 5 and
Table 1 and in accordance with previously reported data, ${ }^{10}$ in carotid atheromatous plaques an intense increase in the immunoreactivity for ICAM-1 was detected. Interestingly, in semiserial sections we also observed increased p53 accumulation within the nuclei of foam cells, as previously reported..$^{33}$ The enhanced expression of MDM2, a classical p53target protein, in the same nuclei strongly suggests that p53 is transcriptionally active in these cells (Table 1). A similar expression pattern was obtained for the p53-target gene $p 21^{W A F 1}$, the latter being also a 'hallmark' of cellular senescence. ${ }^{3}$ Subsequently, we tested in semiserial sections for the presence of senescent cells by using SA- $\beta$-Gal staining and we 
508
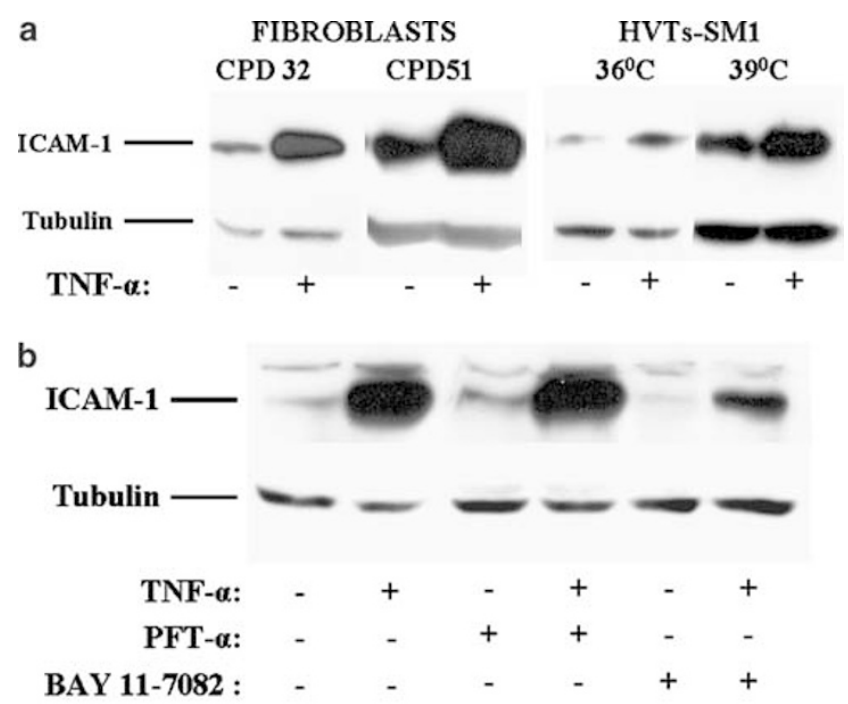

C

\section{FIBROBLASTS HVTs-SM1}

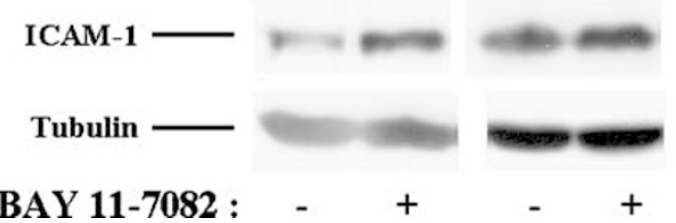

Figure 4 ICAM-1 expression in senescent cells is not mediated by NF- $\kappa$ B. ICAM-1 expression was monitored by Western analysis $24 \mathrm{~h}$ after stimulation with TNF- $\alpha$ in (a) young (CPD 32) and senescent (CPD 51) human fibroblasts, as well as HVTs-SM1 cells at the 36 and $39^{\circ} \mathrm{C}$, and in (b) young fibroblasts (CPD 32) pretreated with BAY 11-7082 or pifithrin- $\alpha$. (c) ICAM-1 expression in senescent human fibroblasts and HVTs-SM1 cells treated for $24 \mathrm{~h}$ with BAY 11-7082.

observed an increased number of positive cells (with perinuclear blue staining) (Table 1). Interestingly, this percentage is even more enhanced in the ICAM1-rich areas of the lesions (Table 1). By contrast, in normal arteries, as expected, we found little ICAM-1 staining and only occasionally p53 activation, whereas no SA- $\beta$-Gal-positive cells were observed (Table 1). Therefore, our data indicate the presence of a subpopulation of senescent cells in the atheromatous plaques, characterized by the coexpression of p53 and ICAM-1.

\section{Discussion}

One of the principal differences between normal and tumor cells is that only the former exhibit a limited proliferative lifespan, while the latter are immortalized, that is, they can proliferate indefinitely. ${ }^{1-3,34}$ This led to the interpretation that the biological role of cellular senescence is a mechanism for restricting tumor progression. ${ }^{34}$ However, several lines of evidence support also an analogy between senescence and aging in vivo. For example, numerous cell types such as epithelial cells, osteoblasts, chondrocytes or smooth muscle cells, when isolated from older donors have very limited or no proliferative capacity. ${ }^{35}$ In addition, cells derived from patients suffering from progeroid syndromes, such as Werner syndrome, exhibit a significantly shorter proliferative lifespan, compared to cells originating from normal donors. ${ }^{36}$ For both biological processes the transcriptional activator p53 seems to be of paramount importance. The role of p53 as a tumor suppressor is well established: nearly $80 \%$ of human cancers display defects in p53 signalling, while half of all cancers harbor structural alterations in the p53 gene..$^{20,37} \mathrm{On}$ the other hand, in senescent cells-characterized by their inability to proliferate-although the levels of p53 protein are not elevated, its DNA-binding and transcriptional activation capacity is highly enhanced. ${ }^{38}$ Moreover, the increased expression of a classical p53-target gene, p21 WAF1 a cyclin-dependent kinase inhibitor, is a 'hallmark' of the initiation of the senescence programme. ${ }^{39}$ Finally, it has been recently shown that mice expressing a hyperactive form of p53 are (as expected) resistant to spontaneous tumorigenesis, but they also develop prematurely several phenotypes associated with normal aging (eg osteoporosis, sarcopenia and skin atrophy, reduced body mass, impaired wound healing, reduced stress tolerance and depletion of hematopoietic stem cells) and their lifespan is approx. $20 \%$ shorter, thus implicating p53 in organismal aging. ${ }^{21}$ These findings are in concert with the hypothesis of 'antagonistic pleiotropy', which predicts that some genes that have been selected during evolution to ensure the maintenance of homeostasis (like cancer prevention) in young organisms, can have deleterious effects in aged individuals. ${ }^{40,41}$

To fully realize the connection between cellular senescence and aging in vivo, it must be mentioned that the senescence phenotype is not restricted to the exhaustion of the proliferative potential. Classical biochemical approaches, as well as, cDNA microarray analyses unraveled that senescent cells are characterized by a less fibrogenic and a pronounced inflammatory phenotype, ${ }^{7,8}$ probably contributing to the aging process. One of the molecules that have been found to be overexpressed in several cell types during senescence, as well as in aged tissues is ICAM-1. ${ }^{6,7}$ The latter is an adhesion molecule implicated in vital aspects of the immune response, ${ }^{9}$ and a well-established NF- $\kappa$ B target. ${ }^{15}$ Nevertheless, we have recently shown that ICAM-1 can also be induced by p53 in an NF- $\kappa$ B-independent manner and thus we hypothesized that a p53ICAM-1 pathway may operate in senescent cells. ${ }^{19}$ Indeed, the data presented here support this hypothesis: we demonstrated that in senescent human cells ICAM-1 is overexpressed at the mRNA and protein levels in parallel with the classical p53target $p 21^{\text {WAF1 }}$ (Figures 1 and 2), and moreover, this 


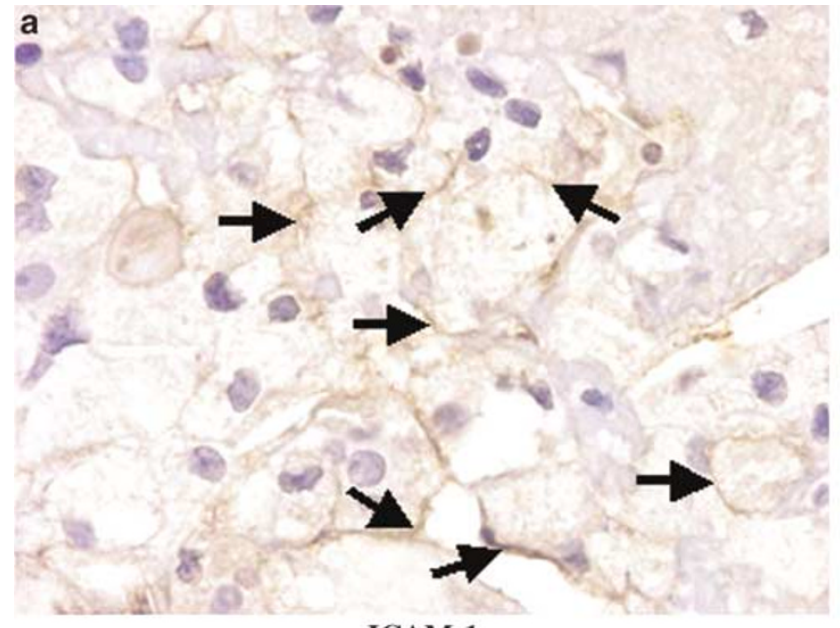

ICAM-1

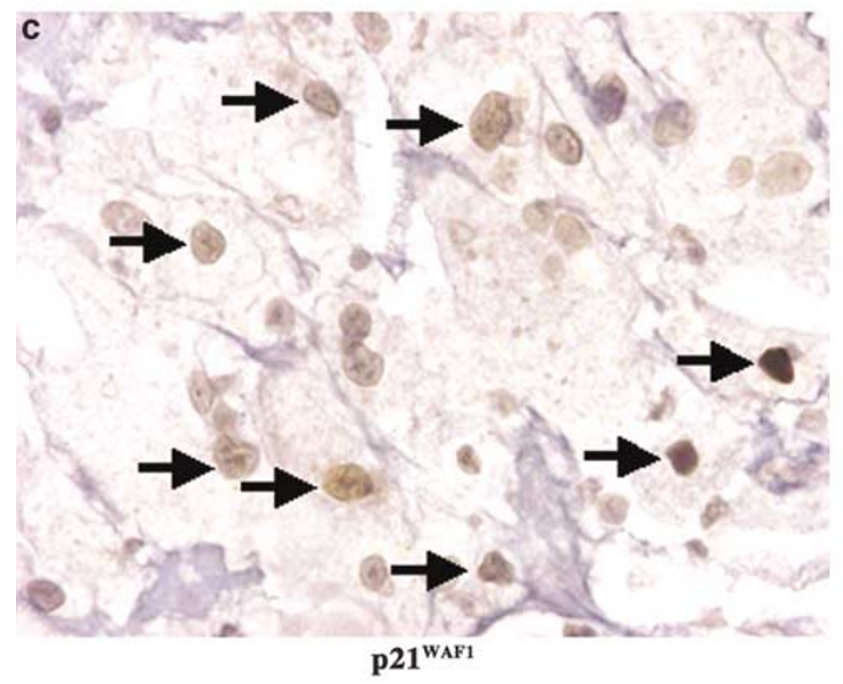

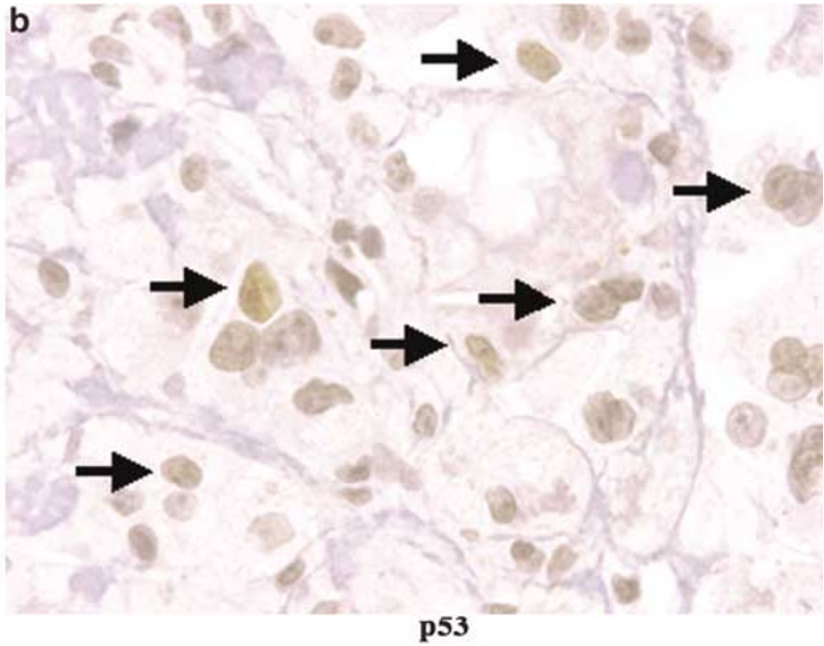

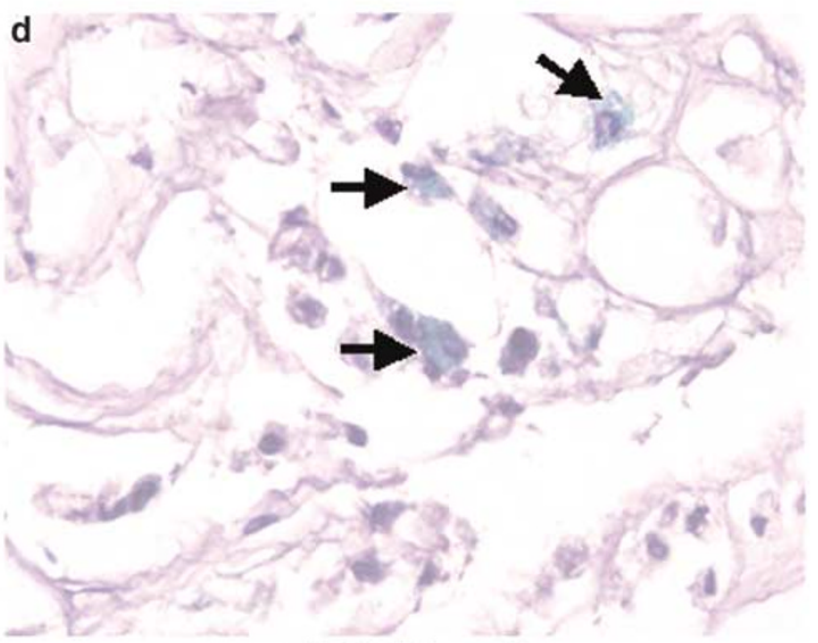

SA- $\beta$-Galactosidase

Figure 5 Identification of p53, p21 ${ }^{\mathrm{WAF} 1}$, ICAM-1 and SA- $\beta$-Gal positive cells in semiserial sections of atheroscletotic lesions. (a) The foam cells exhibit positive anti-ICAM-1 (membranous) staining (arrows). In the same region, colocalization of p53 and p21 ${ }^{\mathrm{WAF} 1}$ (nuclear immunoreactivity, see arrows) in the aforementioned cells is shown (b and $\mathbf{c}$, respectively). The foam cells undergoing senescence are depicted by the positive cytoplasmic SA- $\beta$-Gal staining, indicated by arrows $(\mathbf{d})$. (magnification $\times 400$ ).

Table 1 Percentages of positive cells for p53, p21 ${ }^{\mathrm{WAF} 1}$, MDM2, ICAM-1 and SA- $\beta$-Gal in the atheromatous lesions and controls

\begin{tabular}{|c|c|c|}
\hline & $\begin{array}{l}\text { Atheromatous } \\
\text { lesions ( } \pm \text { s.d.) }\end{array}$ & $\begin{array}{l}\text { Controls } \\
( \pm \text { s.d.) }\end{array}$ \\
\hline p53 & $69.2( \pm 7.4)$ & $0.3( \pm 0.4)$ \\
\hline $\mathrm{p} 21^{\mathrm{WAF} 1}$ & $65.1( \pm 9.3)$ & $3.2( \pm 1.8)$ \\
\hline MDM2 & $58.7( \pm 12.9)$ & $1.0( \pm 1.4)$ \\
\hline ICAM-1 & $32.5( \pm 10.2)$ & $0.5( \pm 0.7)$ \\
\hline SA- $\beta$-gal & $13.6( \pm 5.8)$ & 0.0 \\
\hline $\begin{array}{l}\mathrm{SA}-\beta \text {-gal in the ICAM-1-rich } \\
\text { region }\end{array}$ & $37.6( \pm 7.3)$ & 0.0 \\
\hline
\end{tabular}

overexpression is reversed in a dose-dependent manner by the p53-inhibitor PFT- $\alpha$ (Figures 1-3) but not by the NF- $\kappa$ B inhibitor, BAY 11-7082 (Figure 4). Hence, it seems that p53 activation is responsible for this aspect of the proinflammatory phenotype of senescent cells. Notably, we demonstrated this functional link in two human cellular systems, fibroblasts and smooth muscle cells, the latter being of major importance in the development of atherosclerosis. ${ }^{42}$

Atherosclerosis is an age-related chronic inflammatory disease. ${ }^{43}$ Immunocytochemical studies have revealed an ICAM-1 overexpression in human atherosclerotic plaques. ${ }^{10}$ Although the absence of p53 accelerates atherosclerosis by increasing cell proliferation in apoE-knockout mice, ${ }^{44}$ it has been shown that wild-type p53 accumulates in human atherosclerotic tissues. ${ }^{33,45}$ Moreover, and in agreement with the results presented here (Figure 5, Table 1), it has been reported that the majority of these p53 immunoreactive cells express p21 ${ }^{\mathrm{WAF} 1}{ }^{45}$ These cells are not proliferating, while only few of them exhibit 
the nuclear morphology of apoptosis. ${ }^{33,45}$ The above, in conjunction with our previous observation that ICAM-1 can be induced by p53, ${ }^{19}$ suggest a mechanism for the direct involvement of p53 in atherogenesis. Moreover, aging is a major risk for the development of vascular diseases, such as atherosclerosis. In accordance, we document the presence of a subset of senescent cells in the atherosclerotic lesions, coexpressing activated p53 and ICAM-1, indicating another involvement of p53 in this disorder. This is in concert with previous data showing that human plaque-derived vascular smooth muscle cells (VSMCs) exhibit in culture lower levels of proliferation and an earlier senescence, because of a defect in retinoblastoma protein phosphorylation, the latter being regulated by p53. ${ }^{46}$ In this route, it has been shown that repeated balloon catheter denudation of carotid arteries in experimental animals can lead to accumulation of senescent VSMCs in the neointima and in the media. This is most likely the result of a denudation-induced proliferation in the injured area. ${ }^{47}$ These observations are in symphony with the aforementioned hypothesis of 'antagonistic pleiotropy'. In this context, the p53-mediated ICAM-1 expression reported here may be a paradigm of a beneficial stress response that activates immune reaction; however, the continuous ICAM-1 overexpression can contribute to age-related pathologies, such as atherosclerosis. A similar involvement of 'antagonistically pleiotropic' mechanisms has been proposed also for several other age-related diseases, such as benign and malignant prostate hypertrophy, Alzheimer's disease or even the reciprocal relationship between cellular senescence and carcinogenesis. ${ }^{48,49}$

In conclusion, we report here that p53 activation in senescent cells is responsible for an enhanced expression of ICAM-1, which can play a role in certain age-related aberrations.

\section{Acknowledgements}

This work was partly supported by the European Union (Contract No. QLK6-CT-2002-02582) and the IPE Cyprus (41/2001).

\section{References}

1 Hayflick L. The limited in vitro lifetime of human diploid cell strains. Exp Cell Res 1965;37:614-636.

2 Campisi J. Aging and cancer: the double-edged sword of replicative senescence. J Am Geriatr Soc 1997; 45:482-488.

3 Kletsas D. Aging of fibroblasts. In: Kaul SC, Wadhwa R (eds). Aging of Cells In and Outside the Body. Kluwer Academic Publishers: Dordrecht, The Netherlands, 2003, pp 27-46.

4 Dimri GP, Lee X, Basile G, et al. A biomarker that identifies senescent human cells in culture and in aging skin in vivo. Proc Natl Acad Sci USA 1995;92:9363-9367.

5 Kurz DJ, Decary S, Hong Y, et al. Senescenceassociated (beta)-galactosidase reflects an increase in lysosomal mass during replicative ageing of human endothelial cells. J Cell Sci 2000;113(Part 20): 3613-3622.

6 Minamino T, Miyauchi H, Yoshida T, et al. Endothelial cell senescence in human atherosclerosis: role of telomere in endothelial dysfunction. Circulation 2002; 105:1541-1544.

7 Shelton DN, Chang E, Whittier PS, et al. Microarray analysis of replicative senescence. Curr Biol 1999;9: 939-945.

8 Schnabl B, Purbeck CA, Choi YH, et al. Replicative senescence of activated human hepatic stellate cells is accompanied by a pronounced inflammatory but less fibrogenic phenotype. Hepatology 2003;37:653-664.

9 Cotran RS, Mayadas-Norton T. Endothelial adhesion molecules in health and disease. Pathol Biol (Paris) 1998;46:164-170.

10 Poston RN, Haskard DO, Coucher JR, et al. Expression of intercellular adhesion molecule-1 in atherosclerotic plaques. Am J Pathol 1992;140:665-673.

11 van de Stolpe A, van der Saag PT. Intercellular adhesion molecule-1. J Mol Med 1996;74:13-33.

12 Burne MJ, Elghandour A, Haq M J, et al. IL-1 and TNF independent pathways mediate ICAM-1/VCAM-1 upregulation in ischemia reperfusion injury. J Leukoc Biol 2001;70:192-198.

13 Arnould T, Michiels C, Remacle J. Increased PMN adherence on endothelial cells after hypoxia: involvement of PAF, CD18/CD11b, and ICAM-1. Am J Physiol 1993;264:C1102-C1110.

14 Quarmby S, Kumar P, Kumar S. Radiation-induced normal tissue injury: role of adhesion molecules in leukocyte-endothelial cell interactions. Int J Cancer 1999;82:385-395.

15 Roebuck KA, Finnegan A. Regulation of intercellular adhesion molecule-1 (CD54) gene expression. J Leukoc Biol 1999;66:876-888.

16 Takizawa K, Kamijo R, Ito D, et al. Synergistic induction of ICAM-1 expression by cisplatin and 5 -fluorouracil in a cancer cell line via a NF-kappa B independent pathway. Br J Cancer 1999;80:954-963.

17 Sun B, Fan H, Honda T, et al. Activation of NF-kappa B and expression of ICAM-1 in ischemic-reperfused canine myocardium. J Mol Cell Cardiol 2001;33: 109-119.

18 Hallahan DE, Virudachalam S, Kuchibhotla J. Nuclear factor kappa B dominant negative genetic constructs inhibit X-ray induction of cell adhesion molecules in the vascular endothelium. Cancer Res 1998;58: 5484-5488.

19 Gorgoulis VG, Zacharatos P, Kotsinas A, et al. p53 activates ICAM-1 (CD54) expression in an NF- $\kappa$ B-independent manner. EMBO J 2003;22:15671578.

20 Donehower LA. Does p53 affect organismal aging? J Cell Physiol 2002;192:23-33.

21 Tyner SD, Venkatachalam S, Choi J, et al. p53 mutant mice that display early ageing-associated phenotypes. Nature 2002;415:45-53.

22 Saito H, Papaconstantinou J. Age-associated differences in cardiovascular inflammatory gene induction during endotoxic stress. J Biol Chem 2001;276: 29307-29312. 
23 Pratsinis H, Tsagarakis S, Zervolea I, et al. Chronic in vivo exposure to glucocorticoids prolongs cellular lifespan: the case of Cushing's syndrome-patients' fibroblasts. Exp Gerontol 2002;37:1237-1245.

24 Hsieh JK, Kletsas D, Clunn G, et al. p53, p21(WAF1/ CIP1), and MDM2 involvement in the proliferation and apoptosis in an in vitro model of conditionally immortalized human vascular smooth muscle cells. Arterioscler Thromb Vasc Biol 2000;20:973-981.

25 Pratsinis H, Demoliou-Mason C, Hughes A, et al. A novel in vitro model of conditionally immortalized human vascular smooth muscle cells. A tool for aging studies. Ann NY Acad Sci 2000;908:321-323.

26 Komarov PG, Komarova EA, Kondratov RV, et al. A chemical inhibitor of p53 that protects mice from the side effects of cancer therapy. Science 1999;285: 1733-1737.

27 Pierce JW, Schoenleber R, Jesmok G, et al. Novel inhibitors of cytokine-induced Ikappa Balpha phosphorylation and endothelial cell adhesion molecule expression show anti-inflammatory effects in vivo. J Biol Chem 1997;272:21096-21103.

28 Stary HC, Chandler AB, Dinsmore RE, et al. A definition of advanced types of atherosclerotic lesions and a histological classification of atherosclerosis. A report from the Committee on Vascular Lesions of the Council on Arteriosclerosis, American Heart Association. Circulation 1995;92:1355-1374.

29 Komarova EA, Gudkov AV. Chemoprotection from p53dependent apoptosis: potential clinical applications of the p53 inhibitors. Biochem Pharmacol 2001;62: 657-667.

30 Munro E, Chan P, Patel M, et al. Consistent responses of the human vascular smooth muscle cell in culture: implications for restenosis. J Vasc Surg 1994;20: 482-487.

31 Schwartz SM, Murry CE. Proliferation and the monoclonal origins of atherosclerotic lesions. Annu Rev Med 1998;49:437-460.

32 Manfredi JJ, Prives C. The transforming activity of simian virus 40 large tumor antigen. Biochim Biophys Acta 1994;1198:65-83.

33 Ihling C, Haendeler J, Menzel G, et al. Co-expression of p53 and MDM2 in human atherosclerosis: implications for the regulation of cellularity of atherosclerotic lesions. J Pathol 1998;185:303-312.

34 Wright WE, Shay JW. Cellular senescence as a tumorprotection mechanism: the essential role of counting. Curr Opin Genet Dev 2001;11:98-103.
35 Hornsby PJ. Cellular senescence and tissue aging in vivo. J Gerontol A Biol Sci Med Sci 2002;57: B251-B256.

36 Oshima J, Campisi J, Tannock TC, et al. Regulation of c-fos expression in senescing Werner syndrome fibroblasts differs from that observed in senescing fibroblasts from normal donors. J Cell Physiol 1995;162: 277-283.

37 Lozano G, Elledge SJ. p53 sends nucleotides to repair DNA. Nature 2000;404:24-25.

38 Webley K, Bond JA, Jones CJ, et al. Posttranslational modifications of p53 in replicative senescence overlapping but distinct from those induced by DNA damage. Mol Cell Biol 2000;20:2803-2808.

39 Noda A, Ning Y, Venable SF, et al. Cloning of senescent cell-derived inhibitors of DNA synthesis using an expression screen. Exp Cell Res 1994;211:90-98.

40 Kirkwood TB, Austad SN. Why do we age? Nature 2000;408:233-238.

41 Campisi J. Between Scylla and Charybdis: p53 links tumor suppression and aging. Mech Ageing Dev 2002;123:567-573.

42 Ross R. Atherosclerosis-an inflammatory disease. N Engl J Med 1999;340:115-126.

43 Hansson GK. Immune mechanisms in atherosclerosis. Arterioscler Thromb Vasc Biol 2001;21:1876-1890.

44 Guevara NV, Kim HS, Antonova EI, et al. The absence of p53 accelerates atherosclerosis by increasing cell proliferation in vivo. Nat Med 1999;5:335-339.

45 Ihling C, Menzel G, Wellens E, et al. Topographical association between the cyclin-dependent kinases inhibitor P21, p53 accumulation, and cellular proliferation in human atherosclerotic tissue. Arterioscler Thromb Vasc Biol 1997;17:2218-2224.

46 Bennett MR, Macdonald K, Chan SW, et al. Cooperative interactions between $\mathrm{RB}$ and p53 regulate cell proliferation, cell senescence, and apoptosis in human vascular smooth muscle cells from atherosclerotic plaques. Circ Res 1998;82:704-712.

47 Fenton M, Barker S, Kurz DJ, et al. Cellular senescence after single and repeated balloon catheter denudations of rabbit carotid arteries. Arterioscler Thromb Vasc Biol 2001;21:220-226.

48 Wick G, Berger P, Jansen-Durr P, et al. A Darwinianevolutionary concept of age-related diseases. Exp Gerontol 2003;38:13-25.

49 Krtolica A, Campisi J. Cancer and aging: a model for the cancer promoting effects of the aging stroma. Int J Biochem Cell Biol 2002;34:1401-1414. 